\title{
Fast in-memory XPath search using compressed indexes
}

\author{
Arroyuelo, Diego
}

IEEE Computer Society

2010

Arroyuelo , D , Claude , F , Maneth , S , Mäkinen , V , Navarro , G, Nguyen , K , Sirén , J \& Välimäki , N 2010 , Fast in-memory XPath search using compressed indexes . in ICDE 2010 : 26th IEEE International Conference on Data Engineering . IEEE Computer Society , pp. 417-428, International Conference on Data Engineering, Long Beach, California, United States , 01/03/2010 . https://doi.org/10.1109/ICDE.2010.5447858

http://hdl.handle.net/10138/23556

https://doi.org/10.1109/ICDE.2010.5447858

acceptedVersion

Downloaded from Helda, University of Helsinki institutional repository.

This is an electronic reprint of the original article.

This reprint may differ from the original in pagination and typographic detail.

Please cite the original version. 


\title{
Fast In-Memory XPath Search using Compressed Indexes
}

\author{
Diego Arroyuelo ${ }^{\# 1}$, Francisco Claude ${ }^{* 2}$, Sebastian Maneth ${ }^{+\% 3}$, Veli Mäkinen ${ }^{\dagger 4}$, \\ Gonzalo Navarro $^{\$ 5}$, Kim Nguyễn ${ }^{+6}$, Jouni Sirén ${ }^{\dagger 7}$, Niko Välimäki ${ }^{\dagger 8}$ \\ \#Yahoo! Research Latin America, Chile \\ ${ }^{1}$ darroyueddcc. uchile.cl \\ * David R. Cheriton School of Computer Science, \\ University of Waterloo, Canada \\ ${ }^{2}$ fclaudedcs. uwaterloo.ca \\ ${ }^{+}$NICTA, Australia \\ ${ }^{3}$ sebastian.maneth@nicta.com.au, \\ ${ }^{6} \mathrm{kim} \cdot \mathrm{nguyen} @ \mathrm{nicta} \cdot \mathrm{com} \cdot \mathrm{au}$ \\ ${ }^{\dagger}$ Dept. of Computer Science, University of Helsinki, Finland \\ ${ }_{7}^{4}$ vmakinenecs.helsinki.fi \\ ${ }^{7}$ jltsirenecs.helsinki.fi \\ 8 nvalimak@cs.helsinki.fi \\ $\$$ Dept. of Computer Science, University of Chile, Chile \\ ${ }^{5}$ gnavarroddcc.uchile.cl \\ ${ }^{\%}$ CSE, University of New South Wales, Australia
}

\begin{abstract}
A large fraction of an XML document typically consists of text data. The XPath query language allows text search via the equal, contains, and starts-with predicates. Such predicates can be efficiently implemented using a compressed self-index of the document's text nodes. Most queries, however, contain some parts querying the text of the document, plus some parts querying the tree structure. It is therefore a challenge to choose an appropriate evaluation order for a given query, which optimally leverages the execution speeds of the text and tree indexes. Here the SXSI system is introduced. It stores the tree structure of an XML document using a bit array of opening and closing brackets plus a sequence of labels, and stores the text nodes of the document using a global compressed self-index. On top of these indexes sits an XPath query engine that is based on tree automata. The engine uses fast counting queries of the text index in order to dynamically determine whether to evaluate top-down or bottom-up with respect to the tree structure. The resulting system has several advantages over existing systems: (1) on pure tree queries (without text search) such as the XPathMark queries, the SXSI system performs on par or better than the fastest known systems MonetDB and Qizx, (2) on queries that use text search, SXSI outperforms the existing systems by 1-3 orders of magnitude (depending on the size of the result set), and (3) with respect to memory consumption, SXSI outperforms all other systems for counting-only queries.
\end{abstract}

\section{INTRODUCTION}

As more and more data is stored, transmitted, queried, and manipulated in XML form, the popularity of XPath and XQuery as languages for querying semi-structured data spreads faster. Solving those queries efficiently has proved to be quite challenging, and has triggered much research. Today there is a wealth of public and commercial XPath/XQuery engines, apart from several theoretical proposals.

In this paper we focus on XPath, which is simpler and forms the basis of XQuery. XPath query engines can be roughly divided into two categories: sequential and indexed. In the former, which follows a streaming approach, no preprocessing of the XML data is necessary. Each query must sequentially read the whole collection, and the goal is to be as close as possible to making just one pass over the data, while using as little main memory as possible to hold intermediate results and data structures. Instead, the indexed approach preprocesses the XML collection to build a data structure on it, so that later queries can be solved without traversing the whole collection. A serious challenge of the indexed approach is that the index can use much more space than the original data, and thus may have to be manipulated on disk. There are two approaches for dealing with this problem: (1) to load the index only partially (by using clever clustering techniques), or (2) to use less powerful indexes which require less space. Examples of systems using these approaches are Qizx/DB [1], MonetDB/XQuery [2] and Tauro [3].

In this work we aim at an index for XML that uses little space compared to the size of the data, so that the indexed collection can fit in main memory for moderate-sized data, thereby solving XPath queries without any need of resorting to disk. An in-memory index should outperform streaming approaches, even when the data fits in RAM. Note that usually, main memory XML query systems (such as Saxon [4], Galax [5], Qizx/Open [1], etc.) use machine pointers to represent XML data. We observed that on various well-established DOM implementations, this representation blows up memory consumption to about 5-10 times the size of the original XML document.

An XML collection can be regarded essentially as a text collection (that is, a set of strings) organized into a tree structure, so that the strings correspond to the text data and the tree structure corresponds to the nesting of tags. The problem of manipulating text collections within compressed space is now well understood [6]-[8], and also much work has been carried out on compact data structures for trees (see, e.g., [9] and references therein). In this paper we show how both types of compact data structures can be integrated into a compressed index representation for XML data, which is able to efficiently solve XPath queries. 
A feature inherited from its components is that the compressed index replaces the XML collection, in the sense that the data (or any part of it) can be efficiently reproduced from the index (and thus the data itself can be discarded). The result is called a self-index, as the data is inextricably tied to its index. A self-index for XML data was recently proposed [10], [11], yet its support for XPath is reduced to a very limited class of queries that are handled particularly well.

The main value of our work is to provide the first practical and public tool for compressed indexing of XML data, dubbed Succinct XML Self-Index (SXSI), which takes little space, solves a significant portion of XPath (currently we support at least Core XPath [12], i.e., all navigational axes, plus the three text predicates $=$ (equality), contains, and starts-with), and largely outperforms the best public softwares supporting XPath we are aware of, namely MonetDB and Qizx. The main challenges in achieving our results have been to obtain practical implementations of compact data structures (for texts, trees, and others) that are at a theoretical stage, to develop new compact schemes tailored to this particular problem, and to develop query processing strategies tuned for the specific cost model that emerges from the use of these compact data structures. The limitations of our scheme are that it is inmemory (this is a basic design decision, actually), that it is static (i.e., the index must be rebuilt when the XML data changes), and that it does not handle XQuery. The last two limitations are subject of future work.

\section{BASIC CONCEPTS AND MODEL}

We regard an XML collection as $(i)$ a set of strings and (ii) a labeled tree. The latter is the natural XML parse tree defined by the hierarchical tags, where the (normalized) tag name labels the corresponding node. We add a dummy root so that we have a tree instead of a forest. Moreover, each text node is represented as a leaf labeled \#. Attributes are handled as follows in this model. Each node with attributes is added a single child labeled \&, and for each attribute eattr=value of the node, we add a child labeled attr to its @-node, and a leaf child labeled $\%$ to the att $r$-node. The text content value is then associated to that leaf. Therefore, there is exactly one string content associated to each tree leaf. We will refer to those strings as texts.

Let us call $T$ the set of all the texts and $u$ its total length measured in symbols, $n$ the total number of tree nodes, $\Sigma$ the alphabet of the strings and $\sigma=|\Sigma|, t$ the total number of different tag and attribute names, and $d$ the number of texts (or tree leaves). These receive text identifiers which are consecutive numbers assigned in a left-to-right parsing of the data. In our implementation $\Sigma$ is simply the set of byte values 1 to 255 , and 0 will act as a special terminator called $\$$. This symbol occurs exactly once at the end of each text in $T$. We can easily support multi-byte encodings such as Unicode.

To connect tree nodes and texts, we define global identifiers, which give unique numbers to both internal and leaf nodes, in depth-first preorder. Fig. 1 shows a toy collection (top left) and our model of it (top right), as well as its representation using our data structures (bottom), which serves as a running example for the rest of the paper. In the model, the tree is formed by the solid edges, whereas dotted edges display the connection with the set of texts. We created a dummy root

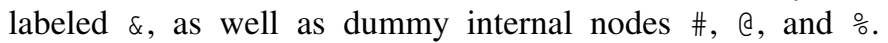
Note how the attributes are handled. There are 6 texts, which are associated to the tree leaves and receive consecutive text numbers (marked in italics at their right). Global identifiers are associated to each node and leaf (drawn at their left). The conversion between tag names and symbols, drawn within the bottom-left component, is used to translate queries and to recreate the XML data, and will not be further mentioned.

Some notation and measures of compressibility follow, preceding a rough description of our space complexities. Logarithms will be in base 2. The empirical $k$-th order entropy [13] of a sequence $S$ over alphabet $\sigma, H_{k}(S) \leq \log \sigma$, is a lower bound to the output size per symbol of any $k$-th order compressor applied to $S$. We will build on self-indexes able of handling text collections $T$ of total length $u$ within $u H_{k}(T)+o(u \log \sigma)$ bits [6], [8], [14]. On the other hand, representing an unlabeled tree of $n$ nodes requires $2 n-O(\log n)$ bits, and several representations using $2 n+o(n)$ bits support many tree query and navigation operations in constant time (e.g., [9]). The labels require in principle other $n \log t$ bits. Sequences $S$ can be stored within $|S| \log \sigma(1+o(1))$ bits (and even $|S| H_{0}(S)+o(|S| \log \sigma)$ ), so that any element $S[i]$ can be accessed, and they can also answer queries $\operatorname{rank}_{c}(S, i)$ (the number of $c$ 's in $S[1, i]$ ) and $\operatorname{select}_{c}(S, j)$ (the position of the $j$-th $c$ in $S$ ) efficiently [14]-[16]. These are essential building blocks for more complex functionalities, as seen later.

The final space requirement of our index will include:

1) $u H_{k}(T)+o(u \log \sigma)$ bits for representing the text collection $T$ in self-indexed form. This supports the string searches of XPath and can (slowly) reproduce any text.

2) $2 n+o(n)$ bits for representing the tree structure. This supports many navigational operations in constant time.

3) $d \log d+o(d \log d)$ bits for the string-to-text mapping, e.g., to determine to which text a string position belongs, or restricting string searches to some texts.

4) Optionally, $u \log \sigma$ or $u H_{k}(T)+o(u \log \sigma)$ bits, plus $O\left(d \log \frac{u}{d}\right)$, to achieve faster text extraction than in 1).

5) $4 n \log t+O(n)$ bits to represent the tags in a way that they support very fast XPath searches.

6) $2 n+o(n)$ bits for mapping between tree nodes and texts.

As a practical yardstick: without the extra storage of texts (item 4) the memory consumption of our system is about the size of the original XML file (and, being a self-index, includes it!), and with the extra store the memory consumption is between 1 and 2 times the size of the original XML file.

In Section III we describe our representation of the set of strings, including how to obtain text identifiers from text positions. This explains items 1,3 , and 4 above. Section IV describes our representation for the tree and the labels, and the way the correspondence between tree nodes and text identifiers works. This explains items 2, 5, and 6. Section V describes how we process XPath queries on top of these compact data 


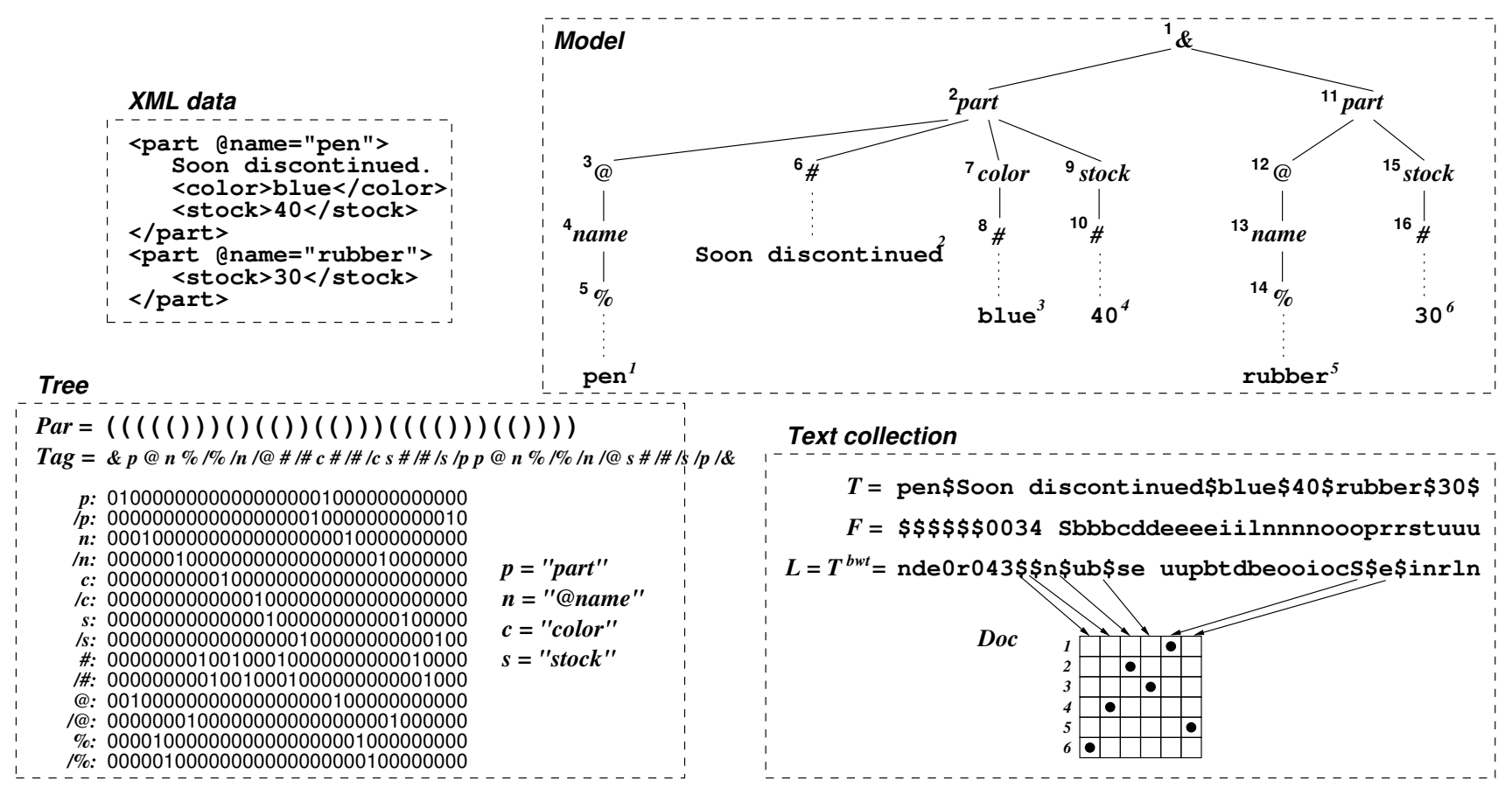

Fig. 1. Our running example on representing an XML collection.

structures. In Section VI we empirically compare our SXSI engine with the most relevant public engines we are aware of.

\section{TEXT REPRESENTATION}

Text data is represented as a succinct full-text self-index [6] that is generally known as the FM-index [17]. The index supports efficient pattern matching that can be easily extended to support different XPath predicates.

\section{A. FM-Index and Backward Searching}

Given a string $T$ of total length $u$, from an alphabet of size $\sigma$, the alphabet-friendly $F M$-index [14] requires $u H_{k}(T)+$ $o(u \log \sigma)$ bits of space. The index supports counting the number of occurrences of a pattern $P$ in $O(|P| \log \sigma)$ time. Locating the occurrences takes extra $O\left(\log ^{1+\epsilon} u\right)$ time per answer, for any constant $\epsilon>1$.

The FM-index is based on the Burrows-Wheeler transform (BWT) of string $T$ [18]. Assume $T$ ends with the special endmarker $\$$. Let $\mathcal{M}$ be a matrix whose rows are all the cyclic rotations of $T$ in lexicographic order. The last column $L$ of $\mathcal{M}$ forms a permutation of $T$ which is the BWT string $L=T^{b w t}$. The matrix is only conceptual; the FM-index uses only on the $T^{b w t}$ string. See Fig. 1 (bottom right). Note $L[i]$ is the symbol preceding the $i$-th lexicographically smallest row of $\mathcal{M}$.

The resulting permutation is reversible. The first column of $\mathcal{M}$, denoted $F$, contains all symbols of $T$ in lexicographic order. There exists a simple last-to-first mapping from symbols in $L$ to $F$ [17]: Let $C[c]$ be the total number of symbols in $T$ that are lexicographically less than $c$. Now the LF-mapping can be defined as $L F(i)=C[L[i]]+\operatorname{rank}_{L[i]}(L, i)$. The symbols of $T$ can be read in reverse order by starting from the end-marker location $i$ and applying $L F(i)$ recursively: we get
$T^{b w t}[i], T^{b w t}[L F(i)], T^{b w t}[L F(L F(i))]$ etc. and finally, after $u$ steps, get the first symbol of $T$. The values $C[c]$ can be stored in a small array of $\sigma \log u$ bits. Function $\operatorname{rank}_{c}(L, i)$ can be computed in $O(\log \sigma)$ time with a wavelet tree data structure requiring only $u H_{k}(T)+o(u \log \sigma)$ bits [14], [15].

Pattern matching is supported via backward searching on the BWT [17]. Given a pattern $P[1, m]$, the backward search starts with the range $[s p, e p]=[1, u]$ of rows in $\mathcal{M}$. At each step $i \in\{m, m-1, \ldots, 1\}$ of the backward search, the range $[s p, e p]$ is updated to match all rows of $\mathcal{M}$ that have $P[i, m]$ as a prefix. New range $\left[s p^{\prime}, e p^{\prime}\right]$ is given by $s p^{\prime}=C[P[i]]+$ $\operatorname{rank}_{P[i]}(L, s p-1)+1$ and $e p^{\prime}=C[P[i]]+\operatorname{rank}_{P[i]}(L, e p)$. Each step takes $O(\log \sigma)$ time [14], and finally ep $-s p+1$ gives the number of times $P$ occurs in $T$.

To find out the location of each occurrence, the text is traversed backwards from each $s p \leq i \leq s p$ (virtually, using $L F$ on $T^{b w t}$ ) until a sampled position is found. This is a sampling carried out at regular text positions, so that the corresponding positions in $T^{b w t}$ are marked in a bitmap $B_{s}[1, u]$, and the text position corresponding to $T^{b w t}[i]$, if $B_{s}[i]=1$, is stored at a samples array $P_{s}\left[\operatorname{rank}_{1}\left(B_{s}, i\right)\right]$. If every $l$-th position of $T$ is sampled, the extra space is $O((n / l) \log n)$ (including the compressed $\left.B_{s}[19]\right)$ and the locating takes $O(l \log \sigma)$ time per occurrence. Using $l=\Theta\left(\log ^{1+\epsilon} u / \log \sigma\right)$ yields $o(u \log \sigma)$ extra space and $O\left(\log ^{1+\epsilon} u\right)$ locating time.

\section{B. Text Collection and Queries}

The textual content of the XML data is stored as \$terminated strings so that each text corresponds to one string. Let $T$ be the concatenated sequence of $d$ texts. The sampling is extended to include all text beginning positions, and to record 
both the text identifier and the offset inside it. Since there are several $\$$ 's in $T$, we fix a special ordering such that the end-marker of the $i$-th text will appear at $F[i]$ in $\mathcal{M}$ (see Fig. 1, bottom right). This generates a valid $T^{b w t}$ of all the texts and makes it easy to extract the $i$-th text starting from its $\$$-terminator. The type of wavelet tree actually used was a Huffman-shaped one using uncompressed bitmaps inside [20].

Now $T^{b w t}$ contains all end-markers in some permuted order. This permutation is represented with a data structure Doc, that maps from positions of $\$ s$ in $T^{b w t}$ to text numbers, and also allows two-dimensional range searching [21] (see Fig. 1, bottom right). Thus the text corresponding to a terminator $T^{b w t}[i]=\$$ is $\operatorname{Doc}\left[\operatorname{rank}_{\$}\left(T^{b w t}, i\right)\right]$. Furthermore, given a range $[s p, e p]$ of $T^{b w t}$ and a range of text identifiers $[x, y]$, $D o c$ can be used to output identifiers of all $\$$-terminators within $[s p, e p] \times[x, y]$ range in $O(\log d)$ time per answer. In practice, because we only use the simpler functionality in the current implementation, Doc is implemented as a plain array using $d \log d$ bits.

The basic pattern matching feature of the FM-index can be extended to support XPath functions such as starts-with, endswith, contains, and operators $=, \leq,<,>, \geq$ for lexicographic ordering. Given a pattern and a range of text identifiers to be searched, these functions return all text identifiers that match the query within the range. In addition, existential (is there a match in the range?) and counting (how many matches in the range?) queries are supported. Time complexities are $O(|P| \log \sigma)$ for the search phase, plus an extra for reporting:

1) $\operatorname{starts-with}(P,[x, y])$ : The goal is to find texts in $[x, y]$ range prefixed by the given pattern $P$. After the normal backward search, the range $[s p, e p]$ in $T^{b w t}$ contains the endmarkers of all the texts prefixed by $P$. Now $[s p, e p] \times[x, y]$ can be mapped to $D o c$, and existential and counting queries can be answered in $O(\log d)$ time. Matching text identifiers can be reported in $O(\log d)$ time per identifier.

2) ends-with $(P,[x, y])$ : Backward searching is localized to texts $[x, y]$ by choosing $[s p, e p]=[x, y]$ as the starting interval. After the backward search, the resulting range $[s p, e p]$ contains all possible matches, thus, existential and counting queries can be answered in constant time. To find out text identifiers for each occurrence, text must be traversed backwards to find a sampled position. Cost is $O(l \log \sigma)$ per answer.

3) operator $=(P,[x, y])$ : texts that are equal to $P$, and in range, can be found as follows. Do the backward search as in ends-with, then map to the $\$$-terminators like in starts-with. Time complexities are same as in starts-with.

4) $\operatorname{contains}(P,[x, y])$ : To find texts that contain $P$, we start with the normal backward search and finish like in ends-with. In this case there might be several occurrences inside one text, which have to be filtered. Thus, the time complexity is proportional to the total number of occurrences, $O(l \log \sigma)$ for each. Existential and counting queries are as slow as reporting queries, but the $O(|P| \log \sigma)$-time counting of all the occurrences of $P$ can still be useful for query optimization.

5) operators $\leq,<,>$, $\geq$ : The operator $\leq$ matches texts that are lexicographically smaller than or equal to the given pattern. It can be solved like the starts-with query, but updating only the $e p$ of each backward search step, while $s p=1$ stays constant. If at some point there are no occurrences of $P[i]=c$ within the prefix $L[1, e p]$, we find those of smaller symbols, $e p=C[c]$, and continue for $P[1, i-1]$. Other operators can be supported analogously, and costs are as for starts-with.

The new XPath extension, XPath Full Text 1.0 [22], suggests a wider selection of functionality for text searching. Implementation of these extensions requires regular expression and approximate searching functionalities, which can be supported within our index using the general backtracking framework [23]: The idea is to alter the backward search to branch recursively to different ranges $\left[s p^{\prime}, e p^{\prime}\right]$ representing the suffixes of the text prefixes (i.e. substrings). This is done by computing $s p_{c}^{\prime}=C[c]+\operatorname{rank}_{c}(L, s p-1)+1$ and $e p_{c}^{\prime}=$ $C[c]+\operatorname{rank}_{c}(L, e p)$ for all $c \in \Sigma$ at each step and recursing on each $\left[s p_{c}^{\prime}, e p_{c}^{\prime}\right]$. Then the pattern (or regular expression) can be compared with all substrings of the texts, allowing to search for approximate occurrences [23]. The running time becomes exponential in the number of errors allowed, but different branch-and-bound techniques can be used to obtain practical running times [24], [25]. We omit further details, as these extensions are out of the scope of this paper.

\section{Construction and Text Extraction}

The FM-index can be built by adapting any BWT construction algorithm. Linear time algorithms exist for the task, but their practical bottleneck is the peak memory consumption. Although there exist general time- and space-efficient construction algorithms, it turned out that our special case of text collection admits a tailored incremental BWT construction algorithm [26] (see the references and experimental comparison therein for previous work on BWT construction): The text collection is split into several smaller collections, and a temporary index is built for each of them separately. The temporary indexes are then merged, and finally converted into a static FM-index.

The BWT allows extracting the $i$-th text by successively applying $L F$ from $T^{b w t}[i]$, at $O(\log \sigma)$ cost per extracted symbol. To enable faster text extraction, we allow storing the texts in plain format in $n \log \sigma$ bits, or in an enhanced LZ78compressed format (derived from the LZ-index [27]) using $u H_{k}(T)+o(u \log \sigma)$ bits. These secondary text representations are coupled with a delta-encoded bit vector storing starting positions of each text in $T$. This bitmap requires $O\left(d \log \frac{u}{d}\right)$ more bits.

\section{TREE REPRESENTATION}

\section{A. Data Representation}

The tree structure of an XML collection is represented by the following compact data structures, which provide navigation and indexed access to it. See Fig. 1 (bottom left).

1) Par: The balanced parentheses representation [28] of the tree structure. This is obtained by traversing the tree in depth-first-search (DFS) order, writing a " (" whenever we arrive at a node, and a ")" when we leave it (thus it is 
easily produced during the XML parsing). In this way, every node is represented by a pair of matching opening and closing parentheses. A tree node will be identified by the position of its opening parenthesis in Par (that is, a node will be just an integer index within Par). In particular, we will use the balanced parentheses implementation of Sadakane [9], which supports a very complete set of operations, including finding the $i$-th child of a node, in constant time. Overall Par uses $2 n+o(n)$ bits. This includes the space needed for constanttime binary rank on Par, which are very efficient in practice.

2) Tag: A sequence of the tag identifiers of each tree node, including an opening and a closing version of each tag, to mark the beginning and ending point of each node. These tags are numbers in $[1,2 t]$ and are aligned with Par so that the tag of node $i$ is simply Tag $[i]$.

We will also need rank and select queries on Tag. Several sequence representations supporting these are known [20]. Given that Tag is not too critical in the overall space, but it is in time, we opt for a practical representation that favors speed over space. First, we store the tags in an array using $\lceil\log 2 t\rceil$ bits per field, which gives constant time access to $\operatorname{Tag}[i]$. The rank and select queries over the sequence of tags are answered by a second structure. Consider the binary matrix $R[1 . .2 t][1 . .2 n]$ such that $R[i, j]=1$ if $\operatorname{Tag}[j]=i$. We represent each row of the matrix using Okanohara and Sadakane's structure sarray [29]. Its space requirement for each row $i$ is $k_{i} \log \frac{2 n}{k_{i}}+k_{i}(2+o(1))$ bits, where $k_{i}$ is the number of times symbol $i$ appears in Tag. The total space of both structures adds up to $2 n \log (2 t)+2 n H_{0}($ Tag $)+n(2+$ $o(1)) \leq 4 n \log t+O(n)$ bits. They support access and select in $O(1)$ time, and rank in $O(\log n)$ time.

\section{B. Tree Navigation}

We define the following operations over the tree structure, which will be useful to support XPath queries over the tree. Most of these operations are supported in constant time, except when a rank over Tag is involved. Let $t a g$ be a tag identifier.

1) Basic Tree Operations: These are direcly inherited from Sadakane's implementation [9]. We mention only the most important ones for this paper; $x$ is a node (a position in Par).

- Close $(x)$ : The closing parenthesis matching Par $[x]$. If $x$ is a small subtree this takes a few local accesses to Par, otherwise a few non-local table accesses.

- Preorder $(x)=\operatorname{rank}_{(}(\operatorname{Par}, i)$ : Preorder number of $x$.

- SubtreeSize $(x)=(\operatorname{Close}(x)-x+1) / 2$ : Number of nodes in the subtree rooted at $x$.

- IsAncestor $(x, y)=x \leq y \leq \operatorname{Close}(x)$ : Whether $x$ is an ancestor of $y$.

- FirstChild $(x)=x+1$ : First child of $x$, if any.

- NextSibling $(x)=$ Close $(x)+1$ : Next sibling of $x$, if any.

- Parent $(x)$ : Parent of $x$. Somewhat costlier than Close $(x)$ in practice, because the answer is less likely to be near $x$ in Par.

2) Connecting to Tags: The following operations are essential for our fast XPath evaluation.
- SubtreeTags $(x, t a g)$ : Returns the number of occurrences of tag within the subtree rooted at node $x$. This is $\operatorname{rank}_{\text {tag }}(\operatorname{Tag}, \operatorname{Close}(x))-\operatorname{rank}_{\text {tag }}(\operatorname{Tag}, x-1)$.

- $\operatorname{Tag}(x)$ : Gives the tag identifier of node $x$. In our representation this is just Tag $[x]$.

- TaggedDesc $(x, t a g)$ : The first node labeled tag strictly within the subtree rooted at $x$. This is select $_{\text {tag }}\left(\right.$ Tag, $\left.\operatorname{rank}_{t a g}(\operatorname{Tag}, x)+1\right)$ if it is $\leq \operatorname{Close}(x)$, and undefined otherwise.

- TaggedPrec $(x, t a g)$ : The last node labeled tag with preorder smaller than that of node $x$, and not an ancestor of $x$. Let $r=\operatorname{rank}_{\text {tag }}(\operatorname{Tag}, x-1)$. If $\operatorname{select}_{t a g}($ Tag, $r)$ is not an ancestor of node $x$, we stop. Otherwise, we set $r=r-1$ and iterate.

- TaggedFoll $(x, t a g)$ : The first node labeled tag with preorder larger than that of $x$, and not in the subtree of $x$. This is $\operatorname{select}_{\text {tag }}\left(\right.$ Tag, $\left.\operatorname{rank}_{\text {tag }}(\operatorname{Tag}, \operatorname{Close}(x))+1\right)$.

3) Connecting the Text and the Tree: Conversion between text numbers, tree nodes, and global identifiers, is easily carried out by using $P a r$ and a bitmap $B$ of $2 n$ bits that marks the opening parentheses of tree leaves containing text, plus $o(n)$ extra bits to support rank/select queries. Bitmap $B$ enables the computation of the following operations:

- LeafNumber $(x)$ : Gives the number of leaves up to $x$ in Par. This is $\operatorname{rank}_{1}(B, x)$.

- TextIds $(x)$ : Gives the range of text identifiers that descend from node $x$. This is simply [LeafNumber $(x-1)+$ 1, LeafNumber $(\operatorname{Close}(x))]$.

- XMLIdText $(d)$ : Gives the global identifier for the text with identifier $d$. This is $\operatorname{Preorder}\left(\operatorname{select}_{1}(B, d)\right)$.

- XMLIdNode $(x)$ : Gives the global identifier for a tree node $x$. This is just $\operatorname{Preorder}(x)$.

\section{Displaying Contents}

Given a node $x$, we want to recreate its text (XML) content, that is, return the string. We traverse the structure starting from $\operatorname{Par}[x]$, retrieving the tag names and the text contents, from the text identifiers. The time is $O(\log \sigma)$ per text symbol (or $O(1)$ if we use the redundant text storage described in Section III) and $O(1)$ per tag.

- $\operatorname{GetText}(d)$ : Generates the text with identifier $d$.

- GetSubtree $(x)$ : Generates the subtree at node $x$.

\section{Handling Dynamic Sets}

During XPath evaluation we need to handle sets of intermediate results, that is, global identifiers. Due to the mechanics of the evaluation, we need to start from an empty set and later carry out two types of operations:

- Insert a new identifier to the result.

- Remove a range of identifiers (actually, a subtree).

To remove a range faster than by brute force, we use a data structure of $2 n-1$ bits representing a perfect binary tree over the interval of global identifiers, so that leaves of this binary tree represent individual positions and internal nodes ranges of positions (i.e., the union of their child ranges). A bit mark 
at each such internal node can be set to zero to implicitly set all its range to zero. A position is in the set if and only if all of its path from the root to it is not zero. Thus one can easily insert elements in $O(\log n)$ time, and remove ranges within the same time, as any range can be covered with $O(\log n)$ binary tree nodes.

\section{XPATH QUERIES}

The aim is to support a practical subset of XPath, while being able to guarantee efficient evaluation based on the data structures described before. As a first shot, we target the "Core XPath" subset [12] of XPath 1.0. It supports all 12 navigational axes, all node tests, and filters with Boolean operations (and, or, not). In our prototype implementation, all axes have been implemented, but only part of the forward fragment (consisting of child and descendant) has been fully optimized. We therefore focus here only on these two axes. A node test (non-terminal NodeTest below) is either the wildcard ('*'), a tag name, or a node type test, i.e., one of text() or node(); the node type tests comment() and processinginstruction() are not supported in our current prototype. Of course, we support all text predicates of XPath 1.0, i.e., the $=$, contains, and starts-with predicates. Here is an EBNF for Core XPath.

$$
\begin{array}{lll}
\text { Core } & ::= & \text { LocationPath | '/ LocationPath } \\
\text { LocationPath } & ::= & \text { LocationStep ('/' LocationStep)* } \\
\text { LocationStep } & ::= & \text { Axis '::' NodeTest } \\
& & \text { | Axis '::' NodeTest '[' Pred ']' } \\
\text { Pred } & ::= & \text { Pred 'and' Pred | Pred 'or' Pred } \\
& & \mid \text { 'not' '(' Pred ')'| Core | '(' Pred ')' }
\end{array}
$$

A data value is the value of an attribute or the content of a text node. Here, all data values are considered as strings. If an XPath expression selects only data values, i.e., its final location step is the attribute-axis or a text() test, then we call it a value expression. Our XPath fragment ("Core+"), consists of Core XPath plus the following data value comparisons which may appear inside filters (that is, may be generated by the nonterminal Pred of above). Let $w$ be a string and $p$ a value expression; if $p$ equals . (dot) or self and the XPath expression to the left of the filter is a value expression, then $p$ is a value expression as well.

- $p=w$ (equality): tests if a string selected by $p$ is equal to $w$.

- contains $(w, p)$ : tests if the string $w$ is contained in a string selected by $p$.

- $\operatorname{starts-with}(p, w)$ : tests if the string $w$ is a prefix of a string selected by $p$.

\section{A. Tree Automata Representation}

It is well-known that Core XPath can be evaluated using tree automata; see, e.g., [30] and [31]. Here we use alternating tree automata (as in [32]). Such automata work with Boolean formulas over states, which must become satisfied for a transition to fire. This allows much more compact representation of queries through automata, than ordinary tree automata (without formulas). Our tree automata work over a binary tree view of

$$
\begin{aligned}
& \overline{\mathcal{R}_{1}, \mathcal{R}_{2}, t^{\prime} \vdash_{\mathcal{A}} \top=(\top, \emptyset)} \text { (true) } \frac{\mathcal{R}_{1}, \mathcal{R}_{2}, t^{\prime} \vdash_{\mathcal{A}} \phi=(b, R)}{\mathcal{R}_{1}, \mathcal{R}_{2}, t^{\prime} \vdash_{\mathcal{A}} \neg \phi=(\bar{b}, \emptyset)} \text { (not) } \\
& \begin{array}{c}
\mathcal{R}_{1}, \mathcal{R}_{2}, t^{\prime} \vdash_{\mathcal{A}} \phi_{1}=\left(b_{1}, R_{1}\right) \\
\frac{\mathcal{R}_{1}, \mathcal{R}_{2}, t^{\prime} \vdash_{\mathcal{A}} \phi_{2}=\left(b_{2}, R_{2}\right)}{\mathcal{R}_{1}, \mathcal{R}_{2}, t^{\prime} \vdash_{\mathcal{A}} \phi_{1} \vee \phi_{2}=\left(b_{1}, R_{1}\right) \otimes\left(b_{2}, R_{2}\right)} \text { (or) }
\end{array} \\
& \mathcal{R}_{1}, \mathcal{R}_{2}, t^{\prime} \vdash_{\mathcal{A}} \phi_{1}=\left(b_{1}, R_{1}\right) \\
& \frac{\mathcal{R}_{1}, \mathcal{R}_{2}, t^{\prime} \vdash_{\mathcal{A}} \phi_{2}=\left(b_{2}, R_{2}\right)}{\mathcal{R}_{1}, \mathcal{R}_{2}, t^{\prime} \vdash_{\mathcal{A}} \phi_{1} \wedge \phi_{2}=\left(b_{1}, R_{1}\right) \otimes\left(b_{2}, R_{2}\right)} \text { (and) } \\
& \frac{q \in \operatorname{dom}\left(\mathcal{R}_{i}\right)}{\mathcal{R}_{1}, \mathcal{R}_{2}, t^{\prime} \vdash_{\mathcal{A}} \downarrow_{i} q=(\top, \mathcal{R}(q))} \text { for } i \in\{1,2\} \text { (left,right) } \\
& \overline{\mathcal{R}_{1}, \mathcal{R}_{2}, t^{\prime} \vdash_{\mathcal{A}} \text { mark }=\left(\top,\left\{t^{\prime}\right\}\right)} \text { (mark) } \\
& \frac{\text { eval_pred }(p)=b}{\mathcal{R}_{1}, \mathcal{R}_{2}, t^{\prime} \vdash \mathcal{A} p=(b, \emptyset)} \text { (pred) } \frac{\text { when no other rule applies }}{\mathcal{R}_{1}, \mathcal{R}_{2}, t^{\prime} \vdash_{\mathcal{A}} \phi=(\perp, \emptyset)}
\end{aligned}
$$

where:

$$
\begin{aligned}
& \bar{\top}=\perp \bar{\perp}=\top
\end{aligned}
$$

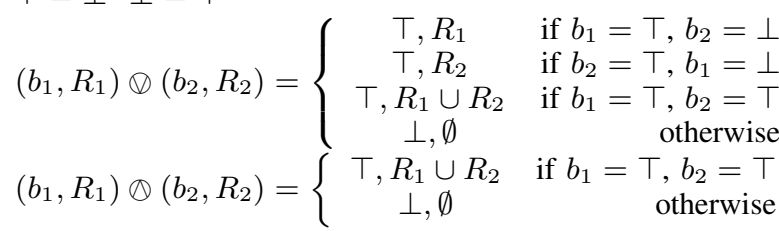

Fig. 2. Inference rules defining the evaluation of a formula

the XML tree where the left child is the first child of the XML node and the right child is the next sibling of the XML node.

Definition 5.1 (Non-deterministic marking automaton): An automaton $\mathcal{A}$ is a tuple $(\mathcal{L}, \mathcal{Q}, \mathcal{I}, \delta)$, where $\mathcal{L}$ is the infinite set of all possible tree labels, $\mathcal{Q}$ is the finite set of states, $\mathcal{I} \subseteq \mathcal{Q}$ is the set of initial states, and $\delta: \mathcal{Q} \times 2^{\mathcal{L}} \rightarrow F$ is the transition function, where $F$ is a set of Boolean formulas. A Boolean formula $\phi$ is generated by the following EBNF.

$$
\begin{array}{llll}
\phi & :=\top|\perp| \phi \vee \phi|\phi \wedge \phi| \neg \phi|a| p & \text { (formula) } \\
a & :=\downarrow_{1} q \mid \downarrow_{2} q & \text { (atom) }
\end{array}
$$

where $p \in P$ is a built-in predicate and $q$ is a state. We call $F$ the set of well-formed formulas.

Definition 5.2 (Evaluation of a formula):

Given an automaton $\mathcal{A}$ and an input tree $t$, the evaluation of a formula is given by the judgement $\mathcal{R}_{1}, \mathcal{R}_{2}, t^{\prime} \vdash_{\mathcal{A}} \phi=(b, R)$

where $\mathcal{R}_{1}$ and $\mathcal{R}_{2}$ are mappings from states to sets of subtrees of $t, t^{\prime}$ is a subtree of $t, \phi$ is a formula, $b \in\{\top, \perp\}$ and $R$ is a set of subtrees of $t$. We define the semantics of this judgment by the mean of inference rules, given in Fig. 2 .

These rules are pretty straightforward and combine the rules for a classical alternating automaton, with the rules of a marking automaton. Rule (or) and (and) implements the Boolean connective of the formula and collect the marking found in their true sub-formulas. Rules (left) and (right) (written as a rule schema for concision) evaluate to true if the state $q$ is in the corresponding set. Intuitively, $\mathcal{R}_{1}$ (resp. $\mathcal{R}_{2}$ ) is 
the set of states accepted in the left (resp. right) subtree of the input tree. Rule (pred) supposes the existence of an evaluation function for built-in predicates. Among the latter, we suppose the existence of a special predicate, mark which evaluates to $T$ and returns the singleton set containing the current subtree. We can now give the semantics of an automaton, by the means of a run function.

\section{Algorithm 5.1 (Top-down run function):}

\section{Input: $\mathcal{A}=(\mathcal{L}, \mathcal{Q}, \mathcal{I}, \delta), t, r \quad$ Output: $\mathcal{R}$}

where $\mathcal{A}$ is the automaton, $t$ the input tree, $r$ a set of states and $\mathcal{R}$ a mapping from states of $\mathcal{Q}$ to sets of subtrees of $t$ and such that $\operatorname{dom}(\mathcal{R}) \subseteq r$.

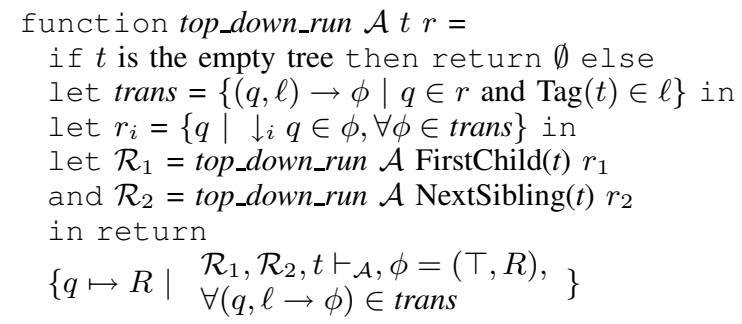

This algorithm works in a very general setting. Considering any subtree $t$ of our input tree, let $\mathcal{R}$ be the result of top_down_run $(\mathcal{A}, t, \mathcal{Q})$. Then $\operatorname{dom}(\mathcal{R})$ is the set of states which accepts $t$ and $\forall q \in \operatorname{dom}(\mathcal{R}), \mathcal{R}(q)$ is the set of subtrees of $t$ marked during a run starting from $q$ on the tree $t$. It is easy to see that the evaluation of top_down_run $(\mathcal{A}, t, r)$ takes time $O(|\mathcal{A}| \times|t|)$, provided that the operations $\oslash, \otimes$ and eval_pred can be evaluated in constant time.

\section{B. From XPath to Automata}

The translation from XPath to alternating automata is simple and can be done in one pass through the parse tree of the XPath expression. Roughly speaking, the resulting automaton is "isomorphic" to the original query (and has approximately the same size). All our optimization discussed later are on-the-fly algorithms; for instance, we only determinize the automaton during its run on the input tree. We illustrate the process by giving a query and its corresponding automaton. Consider the query / descendant: : listitem/descendant: : keyword. The corresponding automaton is $\mathcal{A}=\left(\mathcal{L},\left\{q_{0}, q_{1}\right\},\left\{q_{0}\right\}, \delta\right)$ where $\delta$ contains the following transitions:

$$
\begin{array}{rllll}
1 & q_{0},\{\text { listitem }\} & \rightarrow \downarrow_{1} q_{1} & 4 & q_{1},\{\text { keyword }\} \\
2 & q_{0}, \mathcal{L}-\{\text { mark } \\
3 & q_{0}, \mathcal{L} \quad\{, \#\} & \rightarrow \downarrow_{1} q_{0} & 5 & q_{1}, \mathcal{L}-\{@, \#\} \rightarrow \downarrow_{1} q_{1} \\
& \rightarrow \downarrow_{2} q_{0} & 6 & q_{1}, \mathcal{L} \quad & \rightarrow \downarrow_{2} q_{1}
\end{array}
$$

The automaton starts in state $\left\{q_{0}\right\}$ and traverses the tree until it finds a subtree labeled listitem. At such a subtree, the automaton changes to state $\left\{q_{0}, q_{1}\right\}$ on the left subtree (because it is non-deterministic and two transitions fire), looking for a tag keyword or possibly another tag listitem and it will recurse on the right subtree in state $\left\{q_{0}\right\}$ again. Transitions 2 and 5 make sure that, according to the semantics of the descendant axis, only element nodes (and not text or attributes) are considered. If, in state $\left\{q_{0}, q_{1}\right\}$ it finds a node labeled keyword then this node is marked as a result node.

\section{General Optimizations, On-the-fly Determinisation}

In Algorithm 5.1 the most expensive operation is in Line 11, which is evaluating the set of possible transitions and accumulating the mappings. First, note that only the states outside of filters actually accumulate nodes. All other states always yield empty bindings. Thus we can split the set of states into marking and regular states. This reduces the number of $\emptyset$ and (1) operations on result sets. Note also that given a transition $q_{i}, \ell \rightarrow \downarrow_{1} q_{j} \vee \downarrow_{2} q_{k}$ where $q_{i}, q_{j}$ and $q_{k}$ are marking states, all nodes accumulated in $q_{j}$ are subtrees of the left subtree of the input tree. Likewise, all the nodes accumulated in $q_{k}$ are subtrees of the right subtree of the input tree. Thus both sets of nodes are disjoint. Therefore, we do not need to keep sorted sets of nodes but only need sequences which support $O(1)$ concatenation. Thus, computing the union of two result sets $R_{j}$ and $R_{k}$ can be done in constant time and therefore $\varnothing$ and $\Phi$ can be implemented in constant time.

Another important practical improvement exploits the fact that the automata are very repetitive. For instance if an XPath query does not contain any data value predicate (such as contains) then its evaluation only depends on the tags of the input tree. We can use this to our advantage to memoize the results based on the tag of the input tree and the set $r$. Indeed, the set $r$ and the tag of the input tree $t$ uniquely define the set trans of possible transitions. So instead of computing such a set at every step, we can cache it in a hashtable where the key is the pair $(\operatorname{Tag}(t), r)$; this corresponds to an on-the-fly determinization of automata. We can apply a similar technique for the other expensive operation, that is, the evaluation of the set of formulas. This operation can be split in two parts: the evaluation of the formulas and the propagation of the result sets for the corresponding marking states. Again, if the formulas do not contain data value predicates, then their value only depends on the states present in $\mathcal{R}_{1}$ and $\mathcal{R}_{2}$, the results of the recursive calls. Using the same technique, we can memoize the results in a hash table indexed by the key $\left(\operatorname{dom}\left(\mathcal{R}_{1}\right), \operatorname{dom}\left(\mathcal{R}_{2}\right)\right)$. This hash table contains the pair $\operatorname{dom}(\mathcal{R})$ of the states in the result mapping and a sequence of affectation to evaluate, of the form $\left[q_{i}:=\operatorname{concat}\left(q_{j}, q_{k}\right), \ldots\right]$, which represents results that need to be propagated between the different marking states. Another optimization is for the result set associated with the initial state of the automaton, which is answer of the query. This result set is "final" in the sense that anything that was propagated up to it will be in the result of the query. We can exploit this fact and use a more compact data-structure for this set of results (for instance the one described in Section IVD). Thus we can trade time complexity (since insertion is $O(\log (n))$ in this structure) for space. Using this scheme, we are able to answer queries containing billions of result nodes using little memory.

\section{Leveraging the Speed of the Low-Level Interface}

Conventionally, the run of a tree automaton visits every node of the input tree. This is for instance the behaviour of the tree automata presented in [30], which performs two 
scans of the whole XML document (the latter being stored on disk in a particular format). For highly efficient XPath evaluation, this is not good enough and we must find ways to restrict the run to the nodes that are "relevant" for the query (this is precisely what is also done through "partitioning and pruning" in the staircase join [33]). Consider the query /descendant: : listitem/descendant: : keyword of before. Clearly, we only care about listitem and keyword nodes for this query, and how they are situated with respect to each other. This is precisely the information that is provided through the TaggedDesc and TaggedFoll functions of the tree representation. These functions allow us to have a "contracted" view of the tree, restricted to nodes with certain labels of interest (but preserving the overall tree structure). For instance, to solve the above query we can call TaggedDesc(Root,listitem) which selects the first listitem-node $x$. Now we can apply recursively TaggedDesc(x,keyword) and TaggedFoll(y,keyword) in order to select all keyworddescendants of $x$. We do this optimization of "jumping run" based on the automaton: for a given set of states of the automaton we compute the set of relevant transitions which cause a state change.

Bottom-up run: While the previous technique works well for tree-based queries it still remains slow for value-based queries. For instance, consider the query // listitem//keyword [contains (. , "Unique")]. The text interface described in Section III can answer the string query very efficiently returning the set of text nodes matching this contains query. It is also able to count globally the number of such results. If this number is low, and in particular smaller than the number of listitem or keyword tags in the document (which can also be determined efficiently through the tree structure interface), then it would be faster to take these text nodes as starting point for query evaluation and test if their path to the root matches the XPath expression //listitem//keyword. This scheme is particularly useful for text oriented queries with low selectivity. However, it also applies for tree only queries: imagine the query //listitem//keyword on a tree with many listitem nodes but only a few keyword nodes. We can start bottom-up by jumping to the keyword nodes and then checking their ancestors for listitem nodes.

To achieve this goal, we devise a real bottom-up evaluation algorithm of an automaton. The algorithm takes an automaton and a sequence of potential matching nodes (in our example, the text nodes containing the string "Unique"). It then moves up to the root, using the parent function and checks that the automaton arrives at the root node in its initial state $q_{i}$. The technique used is similar to shift-reduce parsing. Consider a sequence $\left[t_{1}, \ldots, t_{n}\right]$ (ordered in pre-order) of potentially matching subtrees. In our previous example these were text nodes but this is not a necessary condition. The algorithm starts on tree $t_{1}$. First, if the tree is not a leaf, we call the top_down_run function on $t_{1}$ with $r=\mathcal{Q}$. This returns the mapping $\mathcal{R}_{1}$ of all states accepting $t_{1}$. We now want to move from $t_{1}$ upwards to the document root, starting with states $\operatorname{dom}\left(\mathcal{R}_{1}\right)$. Once we arrive at a node $t_{1}^{\prime}$ which is an ancestor of the next potential matching subtree $t_{2}$, we stop at $t_{1}^{\prime}$ and start the algorithm on $t_{2}$ until it reaches $t_{1}^{\prime}$. Once this is done, we can merge both mappings and continue upwards until we reach the root or a common ancestor of $t_{1}^{\prime}$ and $t_{3}$, and so on. The idea of merging the runs at the lowest common ancestor makes sure that we never touch any node more than once, during a bottom-up run. We now give formally the bottom up algorithm.

\section{Algorithm 5.2 (Bottom-up run function):}

\section{Input: $\mathcal{A}, s \quad$ Output: $\mathcal{R}$}

where $\mathcal{A}$ is an automaton, $s$ a sequence of subtrees of the input tree, and $R$ a mapping from states of $\mathcal{A}$ to subtrees of the input tree.

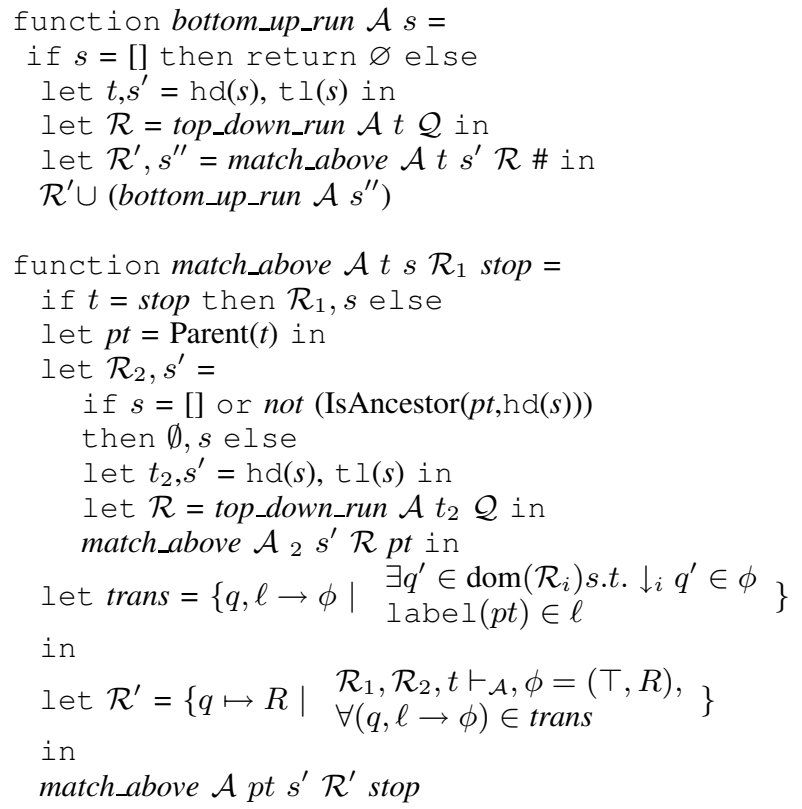

The first function in Algorithm 5.2 iterates the function match_above on every tree in the sequence $s$. The match_above function is the one "climbing-up" the tree. We assume that the Parent(_) function returns the empty tree when applied to the root node. If the input tree is not equal to the tree stop (which is initially the empty tree \#, allowing to stop only after the root node has been processed) then we first check whether the next (we use the function hd and $t 1$ which returns the first element of the list and its tail) potential tree is a descendant of our parent (Line 14). If it is so, then we pause for the current branch and recursively call match_above with our parent as stop tree. Once it returns, we compute all the possible transitions that the automata can take from the parent node to arrive on the left and right subtree with the correct configuration (Line 21). Once this is done, we merge both configuration using the same computation as in the top-down algorithm (Line 23). Finally, we recursively call match_above on the parent node, with the new configuration and sequence of potential matching nodes (Line 25).

\section{EXPERIMENTAL RESUlTS}

We have implemented a prototype XPath evaluator based on the data structures and algorithms presented in previous 
sections. Both the tree structure and the FM-Index were developed in $\mathrm{C}++$, while the XPath engine was written using the Objective Caml language.

\section{A. Protocol}

To validate our approach, we benchmarked our implementation against two other well established XQuery implementations, namely MonetDB/XQuery and Qizx/DB. We describe our experimental settings hereafter.

Test machine: Our test machine features an Intel Core2 Xeon processor at $3.6 \mathrm{Ghz}, 3.8 \mathrm{~GB}$ of RAM and a S-ATA hard drive. The OS is a 64-bit version of Ubuntu Linux. The kernel version is 2.6.27 and the file system used to store the various files is ext3, with default settings. All tests were run on a minimal environment where only the tested program and essential services were running. We used the standard compiler and libraries available on this distribution (namely $\mathrm{g}++4.3 .2$, libxml2 2.6.32 for document parsing and OCaml 3.11.0).

Qizx/DB: We used version 3.0 of Qizx/DB engine (free edition), running on top of the 64-bit version of the JVM (with the -server flag set as recommended in the Qizx user manual). The maximal amount of memory of the JVM set to the maximal amount of physical memory (using the $-\mathrm{Xmx}$ flag). We also used the flag $-r$ of the Qizx/DB command line interface, which allows us to re-run the same query without restarting the whole program (this ensures that the JVM's garbage collector and thread machinery do not impact the performances). We used the timing provided by Qizx debugging flags, and reported the serialization time (which actually includes the materialization of the results in memory and the serialization).

MonetDB/XQuery: We used version Feb2009-SP2 of MonetDB, and in particular, version 4.28.4 of MonetDB4 server and version 0.28 .4 of the XQuery module (pathfinder). We used the timing reported by the "-t" flag of MonetDB client program, mclient. We kept the materialization time and the serialization time separated.

Running times and memory reporting: For each query, we kept the best of five runs. For Qizx/DB, each individual run consists of two repeated runs (" $-r 2$ "), the second one being always faster. For MonetDB, before each batch of five runs, the server was exited properly and restarted. We excluded from the running times the time used for loading the index into main memory (based on the engines timing reports). We monitored the memory the resident set size of each process, which correspond to the amount of process memory actually mapped in physical memory. For MonetDB, we kept track of the memory usage of both server and client. The peak of memory reported was the maximum of the sum of client's memory plus server's memory use, at the same instant.

For the tests where serialization was involved, we serialized to the /dev/null device (that is, all the results were discarded without causing any output operation).

\section{B. Indexing}

Our implementation features a versatile index. It is divided into three parts. First, the tree representation composed of the

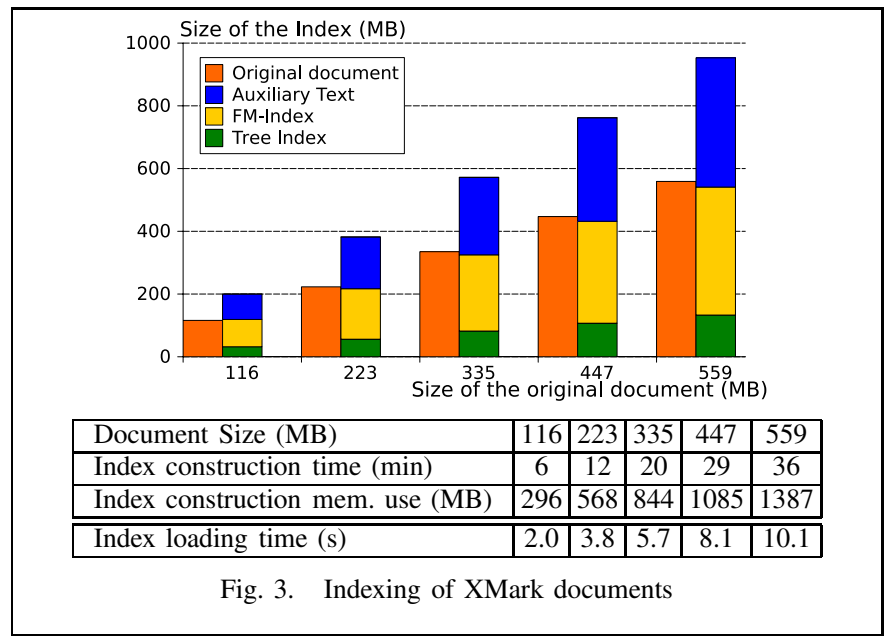

parenthesis structure, as well as the tag structure. Second, the FM-Index encoding the text collection. Third, the auxiliary text representation allowing fast extraction of text content.

It is easy to determine from the query which parts of the index are needed in order to solve it, and thus load only those into main memory. For instance, if a query only involves tree navigation, then having the FM-Index in memory is unnecessary. On the other hand, if we are interested in very selective text-oriented queries, then only the tree part and FM-Index are needed (both for counting and serializing the results). In this case, serialization is a bit slower (due to the cost of text extraction from the FM-Index) but remains acceptable since the number of results is low.

Figure 3 reports the construction time and memory consumption of the indexing process, the loading time from disk into main memory of a constructed index and a comparison between the size of the original document and the size of our in-memory structures. For these indexes, a sampling factor $l=64$ (cf. Section III) was chosen. It should be noted that the size of the tree index plus the size of the FM-index is always less than the size of the original document.

It should be noted that although loading time is acceptable, it dominates query answering time. This is however not a problem for the use case we have targeted: a main memory query engine where the same large document is queried many times. As mentioned in the Introduction, systems such as MonetDB load their indexes only partially; this gives superior performance in a cold-cache scenario than our system.

\section{Tree Queries}

We benchmarked tree queries using the queries given in Fig. 4. Queries Q01 to Q11 were taken from the XPathMark benchmark [34], derived from the XMark XQuery benchmark suite. Q12 to Q16 are "crash tests" that are either simple (Q12 selects only the root since it always has at least one descendant in our files) or generate the same amount of results but with various intermediate result sizes. For this experiment we used XMark documents of size $116 \mathrm{MB}$ and $1 \mathrm{~GB}$. In the cases of MonetDB and Qizx, the files were indexed using 
Q01 /site/regions

Q02/site/closed_auctions

Q03 /site/regions/europe/item/mailbox/mail/text/keyword

Q04/site/closed_auctions/closed_auction/annotation/description/ parlist/listitem

Q05/site/closed_auctions/closed_auction/annotation/description/

Q06 /site/regions/*/item parlist/listitem/parlist/listitem/*//keyword

Q07 //listitem//keyword

Q08 /site/regions/*/item//keyword

Q09 /site/regions/*/person[ address and (phone or homepage) ]

Q10 //listitem[.//keyword and .//emph]//parlist

Q11/site/regions/*/item[ mailbox/mail/date ]/mailbox/mail

Q12/*[ descendant::* ]

$\mathrm{Q} 13 / / *$

$\mathrm{Q} 14 / / * / / *$

$\mathrm{Q} 15 / / * / / * / / * / / *$

$\mathrm{Q} 16 / / * / / * / / * / / * / / * / / * / / * / / *$

Fig. 4. Tree oriented queries

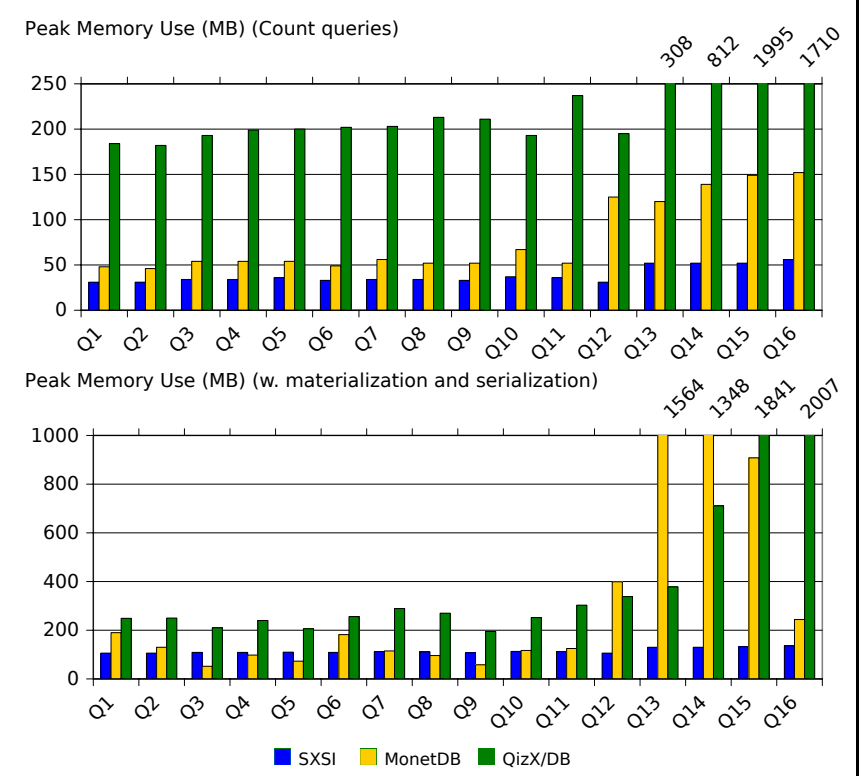

Fig. 6. Peak memory use of the three engines (116 MB XMark file)

the default settings. Fig. 5 reports the running times for both counting and materialization (construction of a result set in memory) and serialization (the output of a result set). As previously mentioned, since Qizx interleaves serialization and materialization, therefore the timing we report include both. In this table, we marked in bold font the lowest materialization time for a given query and we underlined the materialization and serialization time whose sum was the lowest (or in other words underlined numbers correspond to the lowest overall execution time, excluding index loading).

We report in Fig. 6 the peak memory usage for each query, for the $116 \mathrm{MB}$ document.

From the results of Fig. 5, we see how the different components of SXSI contribute to the efficient evaluation model. First, queries Q01 to Q06 — which are fully qualified paths - illustrate the sheer speed of the tree structure and in particular the efficiency of its basic operations (such as FirstChild and NextSibling, which are used for the child axis), as well as the efficient execution scheme provided by the automaton. Query Q07 to Q11 illustrate the impact of the jumping. Moreover, it shows that filters do not impact the execution speed: the conditions they express are efficiently checked by the formula evaluation procedure. Finally, Q12 to Q16 illustrate the robustness of our automata model. Indeed while such queries might seem unrealistic, the good performances that we obtain are only the consequence of using an automata model, which factors in its states all the necessary computation and thus do not materialize unneeded intermediate results. This, coupled together with the compact dynamic set of Section IV-D, allows us to keep a very low memory footprint even when the query returns a lot of results or that each step generates a lot of intermediate results (cf. Fig. 6).

It is well-known that MonetDB's policy is to use as much memory as available to answer queries efficiently and to preserve memory only if there is not enough physical memory available. Our goal by providing memory use experiment was not to argue that we would use less memory than e.g. MonetDB but rather to show that even though we remain memory conscious, we can outperform engines using a "greedier" memory policy.

\section{Text Queries}

We tested the text capabilities of our XPath engine against the most advanced text oriented features of other query engines.

Qizx/DB: We used the newly introduced Full-Text extension of XQuery available in Qizx/DB v. 3.0. We tried to write queries as efficiently as possible while preserving the same semantics as our original queries. The query we used always gave better results than their pure XPath counterpart. In particular, we used the ftcontains text predicate [22] implemented by Qizx/DB. The ftcontains predicate allows one to express not only contains-like queries but also Boolean operations on text predicates, regular expression matching and so on. It is more efficient than the standard contains. In particular we used regular expression matching instead of of the starts-with and ends-with operators since the latter were slower in our experiments.

MonetDB: MonetDB supports some full-text capabilities through the use of the PF/Tijah text index ( [35]). However, this index only supports a complex about operator, similar to contains but returning ranked results by order of relevance. Although its semantics does not exactly match the one of contains, its execution is often faster while providing richer results. We measured MonetDB timings both for standard XPath operator and about.

Experiments were made on a $122 \mathrm{MB}$ Medline file. This file contains bibliographic information about life sciences 


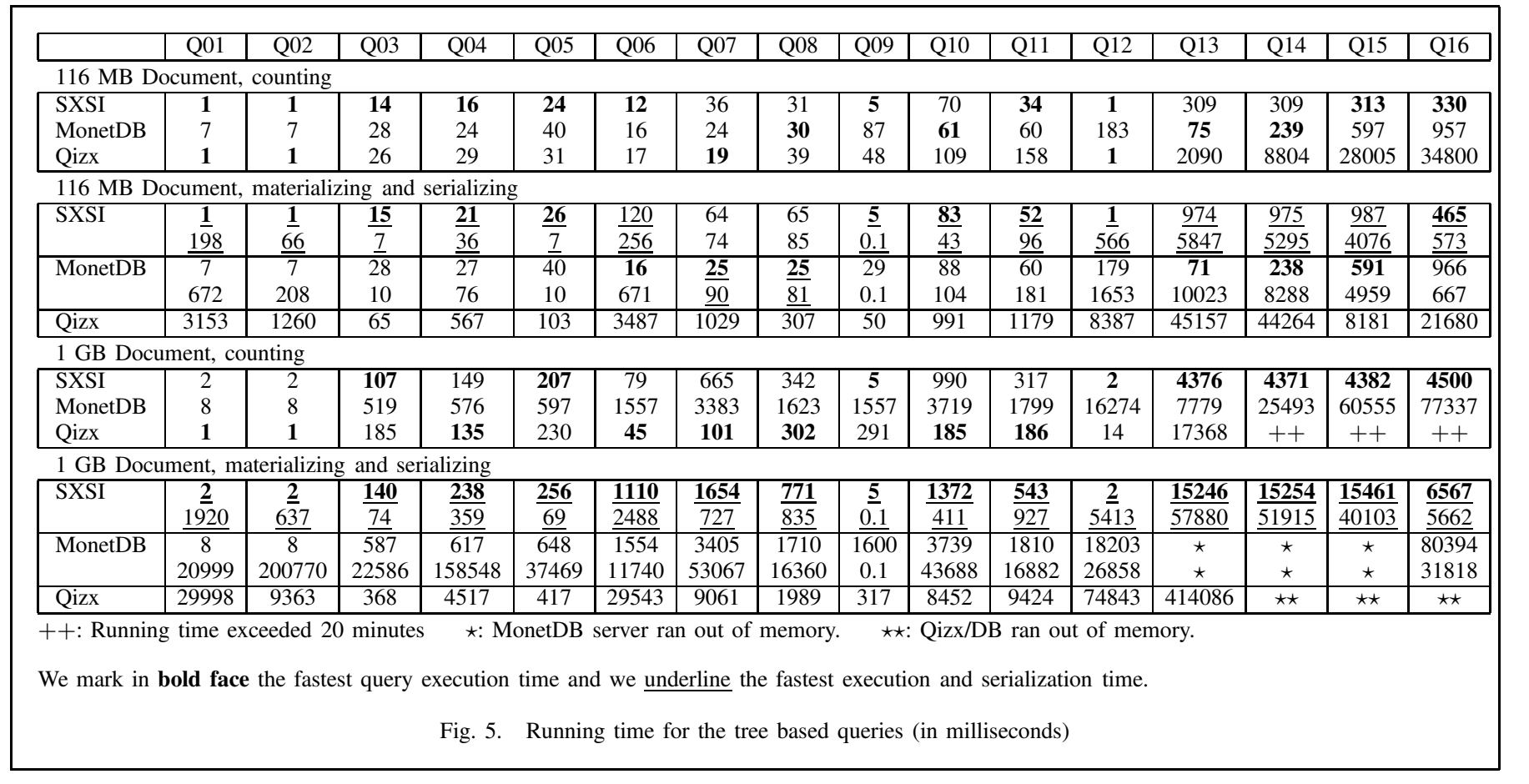

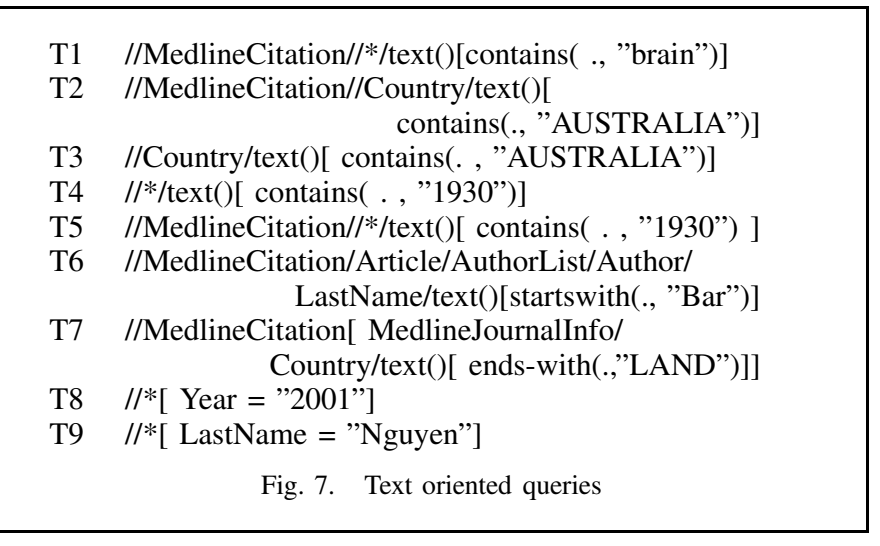

and biomedical publications. This test file featured 5,732,159 text elements, for a total amount of $95 \mathrm{MB}$ of text content. Fig. 7 shows the text queries we tested. We used count queries for both MonetDB and Qizx - enclosing the query in an $\mathrm{fn}$ : count () predicate- while in our implementation we ran the queries in "materialization" mode but without serializing the output. The table in Fig. 8 summarizes the running times for each query. As we target very selective text queries, we also give, for each query, the number of results it returned. Since for these queries our automata worked in "bottom-up" mode, we detail the two following operations:

- Calling the text predicate globally on the text collection, thus retrieving all the probable matches of the query (Text query line in the table of Fig. 8)

- Running the automaton bottom up from the set of probable matches to keep those satisfying the path expression

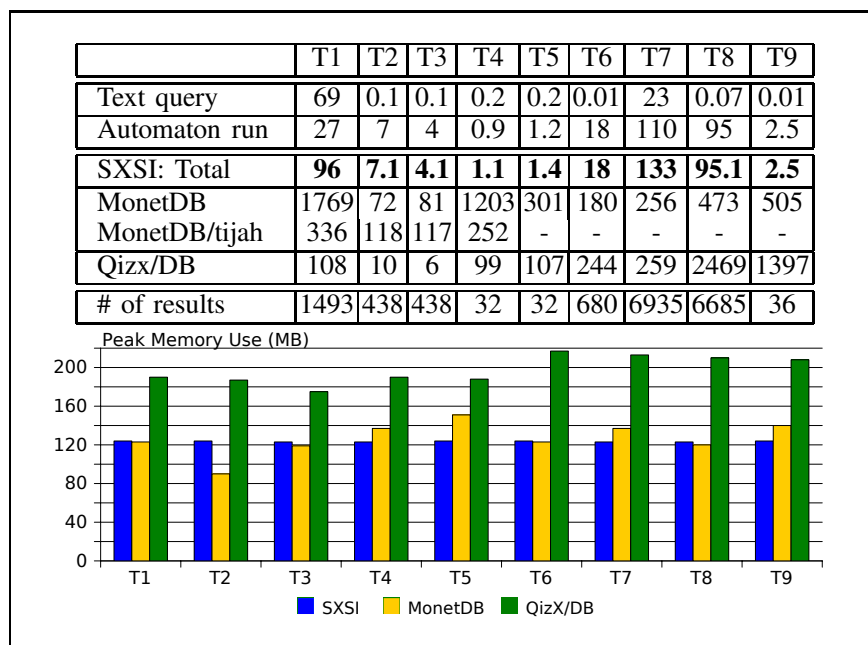

Fig. 8. Running times (in ms) and memory consumption (in MB) for the text-oriented queries

(Automaton run line in the table of Fig. 8)

As it is clear from the experiments the bottom-up strategy pays off. The only down-side of this approach is that the automaton uses Parent moves, which are less efficient than FirstChild and NextSibling. This is clear in queries T7 and T8 where the increase in number of results makes the relative slowness of the automata more visible. However our evaluator still outperforms the other engines even in those cases.

\section{E. Remarks}

We also compared with Tauro [3]. Yet, as it uses a tailored query language, we could not produce comparable results. 
We limited the experiments to natural language XML, although our engine (unlike the inverted file based engines) supports as well queries on XML databases of continuous sequences such as DNA and proteins. Realistic queries on such biosequence XMLs require approximate / regular expression search functionalities, that we already support but whose experimental study is out of the scope of this paper.

\section{CONClusions ANd Future Work}

We have presented SXSI, a system for representing an XML collection in compact form so that fast indexed XPath queries can be carried out on it. Even in its current prototype stage, SXSI is already competitive with well-known efficient systems such as MonetDB and Qizx. As such, a number of avenues for future work are open. We mention the broadest ones here.

Handling updates to the collections is possible in principle, as there are dynamic data structures for sequences, trees, and text collections [7]-[9]. What remains to be verified is how practical can those theoretical solutions be made.

As seen, the compact data structures support several fancy operations beyond those actually used by our XPath evaluator. A matter of future work is to explore other evaluation strategies that take advantage of those nonstandard capabilities. As an example, the current XPath evaluator does not use the range search capabilities of structure Doc of Section III. An interesting challenge is to support XPath string-value semantics, where strings spanning more than one text node can be searched for. This, at least at a rough level, is not hard to achieve with our FM-index, by removing the \$-terminators and marking them on a separate bitmap instead. Beyond that, we would like to extend our implementation to full XPath 1.0, and add core functionalities of XQuery.

\section{ACKNOWLEDGEMENTS}

We would like to thank Schloss Dagstuhl for the very pleasant and stimulating research environment it provides; the work of this paper was initiated during the Dagstuhl seminar "Structure-Based Compression of Complex Massive Data" (Number 08261). Diego Arroyuelo and Francisco Claude were partially funded by NICTA, Australia. Francisco Claude was partially funded by NSERC of Canada and the Go-Bell Scholarships Program. Francisco Claude and Gonzalo Navarro were partially funded by Fondecyt Grant 1-080019, Chile. Gonzalo Navarro was partially funded by Millennium Institute for Cell Dynamics and Biotechnology (ICDB), Grant ICM P05-001-F, Mideplan, Chile. Veli Mäkinen and Jouni Sirén were funded by the Academy of Finland under grant 119815. Niko Välimäki was funded by the Helsinki Graduate School in Computer Science and Engineering.

\section{REFERENCES}

[1] XML Mind products, "Qizx XML query engine," http://www.xmlmind.com/qizx, 2007.

[2] P. A. Boncz, T. Grust, M. van Keulen, S. Manegold, J. Rittinger, and J. Teubner, "MonetDB/XQuery: a fast XQuery processor powered by a relational engine," in SIGMOD, 2006, pp. 479-490.

[3] Signum, "Tauro," http://tauro.signum.sns.it/, 2008.
[4] M. Kay, "Ten reasons why Saxon XQuery is fast," IEEE Data Eng. Bull., vol. 31, no. 4, pp. 65-74, 2008.

[5] M. F. Fernández, J. Siméon, B. Choi, A. Marian, and G. Sur, "Implementing XQuery 1.0: The Galax experience," in $V L D B, 2003$, pp. $1077-1080$.

[6] G. Navarro and V. Mäkinen, "Compressed full-text indexes," $A C M$ Comp. Surv., vol. 39, no. 1, 2007.

[7] H.-L. Chan, W.-K. Hon, T.-W. Lam, and K. Sadakane, "Compressed indexes for dynamic text collections," ACM TALG, vol. 3, no. 2, 2007.

[8] V. Mäkinen and G. Navarro, "Dynamic entropy-compressed sequences and full-text indexes," ACM TALG, vol. 4, no. 3, 2008.

[9] K. Sadakane and G. Navarro, "Fully-functional static and dynamic succinct trees," in SODA, 2010.

[10] P. Ferragina, F. Luccio, G. Manzini, and S. Muthukrishnan, "Structuring labeled trees for optimal succinctness, and beyond," in FOCS, 2005, pp. 184-196.

[11] _ "Compressing and searching XML data via two zips," in $W W W$, 2006, pp. 751-760.

[12] G. Gottlob, C. Koch, and R. Pichler, "Efficient algorithms for processing XPath queries," ACM TODS, vol. 30, no. 2, pp. 444-491, 2005.

[13] G. Manzini, "An analysis of the Burrows-Wheeler transform," J. ACM, vol. 48, no. 3, pp. 407-430, 2001.

[14] P. Ferragina, G. Manzini, V. Mäkinen, and G. Navarro, "Compressed representations of sequences and full-text indexes," $A C M T A L G$, vol. 3, no. 2, 2007.

[15] R. Grossi, A. Gupta, and J. S. Vitter, "High-order entropy-compressed text indexes," in SODA, 2003, pp. 841-850.

[16] A. Golynski, I. Munro, and S. Rao, "Rank/select operations on large alphabets: a tool for text indexing," in SODA, 2006, pp. 368-373.

[17] P. Ferragina and G. Manzini, "Indexing compressed text," J. ACM, vol. 54, no. 4, pp. 552-581, 2005.

[18] M. Burrows and D. J. Wheeler, "A block-sorting lossless data compression algorithm." Digital Equipment Corporation, Tech. Rep. 124, 1994.

[19] R. Raman, V. Raman, and S. S. Rao, "Succinct indexable dictionaries with applications to encoding $k$-ary trees and multisets," in SODA, 2002, pp. 233-242.

[20] F. Claude and G. Navarro, "Practical rank/select queries over arbitrary sequences," in SPIRE, 2008, pp. 176-187.

[21] V. Mäkinen and G. Navarro, "Rank and select revisited and extended," Theor. Comput. Sci., vol. 387, no. 3, pp. 332-347, 2007.

[22] "XQuery and XPath Full Text 1.0," http://www.w3.org/TR/xpath-fulltext-10.

[23] T. W. Lam, W. K. Sung, S. L. Tam, C. K. Wong, and S. M. Yiu, "Compressed indexing and local alignment of DNA," Bioinformatics, vol. 24, no. 6, pp. 791-797, 2008

[24] B. Langmead, C. Trapnell, M. Pop, and S. L. Salzberg, "Ultrafast and memory-efficient alignment of short dna sequences to the human genome," Genome Biology, vol. 10, no. 3, 2009, R25.

[25] H. Li and R. Durbin, "Fast and accurate short read alignment with burrows-wheeler transform," Bioinformatics, 2009, advance access.

[26] J. Sirén, "Compressed suffix arrays for massive data," in SPIRE, 2009, pp. 63-74.

[27] D. Arroyuelo, G. Navarro, and K. Sadakane, "Reducing the space requirement of LZ-index," in CPM, 2006, pp. 319-330.

[28] I. Munro and V. Raman, "Succinct representation of balanced parentheses, static trees and planar graphs," in FOCS, 1997, pp. 118-126.

[29] D. Okanohara and K. Sadakane, "Practical entropy-compressed rank/select dictionary," in ALENEX, 2007.

[30] C. Koch, "Efficient processing of expressive node-selecting queries on XML data in secondary storage: a tree automata-based approach," in $V L D B, 2003$, pp. 249-260.

[31] H. Björklund, W. Gelade, M. Marquardt, and W. Martens, "Incremental XPath evaluation," in ICDT, 2009, pp. 162-173.

[32] H. Comon, M. Dauchet, R. Gilleron, F. Jacquemard, C. Löding, D. Lugiez, S. Tison, and M. Tommasi, "Tree automata techniques and applications," http://www.grappa.univ-lille3.fr/tata, 2007.

[33] T. Grust, M. van Keulen, and J. Teubner, "Staircase join: Teach a relational DBMS to watch its (axis) steps," in VLDB, 2003, pp. 524-525.

[34] M. Franceschet, "XPathMark: Functional and performance tests for XPath," in XQuery Implementation Paradigms, 2007, http://drops.dagstuhl.de/opus/volltexte/2007/892.

[35] J. A. List, V. Mihajlovic, G. Ramírez, A. P. de Vries, D. Hiemstra, and H. E. Blok, "TIJAH: Embracing IR methods in XML databases," Inf. Retr., vol. 8, no. 4, pp. 547-570, 2005. 